\title{
Polypharmacy in older patients with chronic diseases: a cross-sectional analysis of factors associated with excessive polypharmacy
}

Anja Rieckert ${ }^{1 *} \mathbb{B}$, Ulrike S. Trampisch ${ }^{2}$, Renate Klaaßen-Mielke², Eva Drewelow ${ }^{3}$, Aneez Esmail $^{4}$, Tim Johansson $^{5}$, Sophie Keller ${ }^{5}$, Ilkka Kunnamo ${ }^{6}$, Christin Löffler ${ }^{3}$, Joonas Mäkinen ${ }^{6}$, Giuliano Piccoliori ${ }^{7}$, Anna Vögele ${ }^{7}$ and Andreas Sönnichsen ${ }^{1,4}$

\begin{abstract}
Background: Polypharmacy is common in older people and associated with potential harms. The aim of this study was to analyse the characteristics of an older multimorbid population with polypharmacy and to identify factors contributing to excessive polypharmacy in these patients.

Methods: This cross-sectional analysis is based on the PRIMA-eDS trial, a large randomised controlled multicentre study of polypharmacy in primary care. Patients' baseline data were used for analysis. A number of socioeconomic and medical data as well as SF-12-scores were entered into a generalized linear mixed model to identify variables associated with excessive polypharmacy (taking $\geq 10$ substances daily).

Results: Three thousand nine hundred four participants were recruited. Risk factors significantly associated with excessive polypharmacy were frailty (OR 1.45; 95\% Cl 1.22-1.71), > 8 diagnoses (OR 2.64; 95\% Cl 2.24-3.11), BMI $\geq 30$ (OR 1.18; 95\% Cl 1.02-1.38), a lower SF-12 physical health composite score (OR 1.47; 95\% Cl 1.26-1.72), and a lower SF-12 mental health composite score (OR 1.33; 95\% Cl 1.17-1.59) than the median of the study population ( $\leq 36.6$ and $\leq 48.7$, respectively). Age $\geq 85$ years (OR $0.83 ; 95 \% \mathrm{Cl} 0.70-0.99$ ) led to a significantly lower risk for excessive polypharmacy. No association with excessive polypharmacy could be found for female sex, low educational level, and smoking. Regarding the study centres, being recruited in the UK led to a significantly higher risk for excessive polypharmacy compared to being recruited in Germany 1/Rostock (OR 1.71; 95\% Cl 1.27-2.30). Being recruited in Germany 2/Witten led to a slightly significant lower risk for excessive polypharmacy compared to Germany $1 /$ Rostock (OR 0.74; 95\% Cl 0.56-0.97).

Conclusions: Frailty, multimorbidity, obesity, and decreased physical as well as mental health status are risk factors for excessive polypharmacy. Sex, educational level, and smoking apparently do not seem to be related to excessive polypharmacy. Physicians should especially pay attention to their frail, obese patients who have multiple diagnoses and a decreased health-related quality of life, to check carefully whether all the drugs prescribed are evidencebased, safe, and do not interact in an unfavourable way.
\end{abstract}

Trial registration: This trial has been registered with Current Controlled Trials Ltd. on 31 July 2014 (ISRCTN10137559).

Keywords: Aged, Risk factors, Protective factors, Polypharmacy, Europe, Cross-sectional study, PRIMA-eDS

\footnotetext{
* Correspondence: Anja.Rieckert@uni-wh.de

${ }^{1}$ Institute of General Practice and Family Medicine, Faculty of Health, Witten/

Herdecke University, Alfred-Herrhausen-Str. 50, 58448 Witten, Germany

Full list of author information is available at the end of the article
}

(c) The Author(s). 2018 Open Access This article is distributed under the terms of the Creative Commons Attribution 4.0 International License (http://creativecommons.org/licenses/by/4.0/), which permits unrestricted use, distribution, and reproduction in any medium, provided you give appropriate credit to the original author(s) and the source, provide a link to the Creative Commons license, and indicate if changes were made. The Creative Commons Public Domain Dedication waiver (http://creativecommons.org/publicdomain/zero/1.0/) applies to the data made available in this article, unless otherwise stated. 


\section{Background}

Older adults ( $\geq 65$ years) make up an increasing proportion of the European population, particularly the oldest old ( $\geq 80$ years) [1], and drug use in this age group is common. Depending on setting and age, older adults are prescribed an average of 5.3-6.9 drugs [2-5]. Around 44.2$57.7 \%$ of older adults are on $\geq 5$ different drugs, and additional 9.1-23.2\% on $\geq 10$ different drugs [4, 6-8]. Concurrent use of multiple medications is known as polypharmacy [9]. However, there is no consensus on the definition of polypharmacy in the literature. Likewise, there is no agreed definition of excessive polypharmacy, though the cut-off point of $\geq 10$ drugs is often used [10].

In some cases, polypharmacy may be inevitable, however, in many patients it appears to be inappropriate [11]. This may be specifically true for older people, as age-related changes in pharmacokinetics and pharmacodynamics increase the risk of adverse drug events [12]. There is some evidence, mostly from observational studies, that polypharmacy in older adults is associated with a number of negative health outcomes such as decreased functional and cognitive health status, increased risk of falls, adverse drug events, hospitalisations, and mortality. However, not all studies found these associations [13]. Risks of adverse drug outcomes increase with an increasing number of medications [14]. Wimmer et al. assessed the association between medication regimen complexity in older people and clinical outcomes, and they concluded that regimen complexity is associated with medication nonadherence and increased rates of hospitalisation [15]. In a retrospective cohort study of adults aged $\geq 20$ years, Payne et al. found an association between unplanned hospital admission and consumption of multiple medications. However, after controlling for multimorbidity only consumption of $\geq 10$ medications was significantly associated with unplanned admissions [16]. Also, the King's Fund has proposed a cut-off of $\geq 10$ medications as a pragmatic approach to identify polypharmacy patients 'at risk', whilst recognising that there is no universal consensus around this [17].

In previous research the association between no-polypharmacy and polypharmacy as well as between no-polypharmacy and excessive polypharmacy was investigated $[4,5,18-25]$. To our knowledge no study so far has analysed possible predictors for excessive polypharmacy in patients consuming multiple drugs. Given the increased risk of adverse health outcomes in older adults taking $\geq 10$ medications it is important to investigate which factors contribute to excessive polypharmacy. We therefore are analysing in this study the independent descriptive variables of the PRIMA-eDS-trial (Polypharmacy in chronic diseases: Reduction of Inappropriate Medication and Adverse drug events in older populations by electronic Decision Support) population to identify possible predictors of excessive polypharmacy (using the cut-off of $\geq 10$ substances as proposed above).

\section{Methods \\ Study design and population}

This cross-sectional study is based on the baseline data of the PRIMA-eDS trial. In 2015, GPs in five study centres (UK/Manchester, Italy/Bolzano, Austria/Salzburg, Germany 1/Rostock, Germany 2/Witten) were enrolled to recruit patients with polypharmacy. Patient eligibility criteria included age $\geq 75$ and taking $\geq 8$ medications regularly. Patient data were collected between September 2014 and September 2015. A more detailed description of the PRIMA-eDS trial has been published [26]. These baseline patient data were used in the analysis presented here.

\section{Data collection}

At each practice the GP or an authorised staff member collected patient data and entered it into an electronic case report form (eCRF; Additional file 1). In the UK, data was also collected at some practices by a regional Clinical Research Network research nurse working with the practice. Baseline data used for this study included: age, sex, height, weight, all drugs with an Anatomical Therapeutic Chemical (ATC) code (prescribed and over the counter), all diagnoses, smoking status, and frailty according to the clinical frailty scale [27]. A health-related quality of life questionnaire (SF-12v2) [28] was also administered to each patient and entered into the eCRF by staff of the study centres. Educational level was recorded according to ISCED-97 which was filled out by the patient [29].

\section{Outcome and independent variables}

According to the King's Fund [17] and the median number of drugs used by all patients participating in the study, we defined the outcome variables as non-excessive polypharmacy $(<10$ substances) and excessive polypharmacy $(\geq 10$ substances). Drugs were coded using the ATC [30] classification system of the World Health Organisation and updated to correspond with changes in 2016 and 2017. Each substance was recorded separately. We took into consideration all substances at baseline with an ATC code. Diagnoses were recorded using the ICD-10 code [31].

Independent variables used in the analysis were age, sex, educational level, physical and mental health composite scores derived from the SF-12, frailty status, body mass index (BMI), smoking status, study centre, and number of diagnoses.

\section{Statistical analysis}

Descriptive statistics were used to describe patient demographics and other variables. Means and standard deviations (SD) were used for continuous variables, and proportions for 
categorical variables. A multivariable generalized linear mixed model was applied to identify factors associated with excessive polypharmacy, with GP practice as a random effect. For the purposes of this analysis, some independent variables were re-categorised: age (into $\geq 85$ years versus $<85$ ); educational level (low versus medium/high); frailty (frail/terminally ill versus managing well/vulnerable); body mass index (BMI $\geq 30 \mathrm{~kg} / \mathrm{m}^{2}$ versus BMI $<30 \mathrm{~kg} / \mathrm{m}^{2}$ ); smoking status (smoker versus non-smoker/ex-smoker). The number of diagnoses was grouped as $>$ median versus $\leq$ median $(>8$ vs $\leq 8$ diagnoses). Physical and mental health composite scores were all grouped as $\leq$ median versus >median, which was for the physical health composite score $\leq 36.635$ versus $>36.636$ and for the mental health composite score $\leq 48.7$ versus $>48.8$. Lower physical and mental health composite scores, meaning that they are below the median, imply poorer health-related quality of life. As some variables have a high share of missing data, we performed multiple imputations by fully conditional specification. Cramer's V was used to look for bivariate associations. A significance level of $\alpha=0.05$ was used throughout. Data was analysed using the SAS v9.4 statistical software.

\section{Results}

Three hundred fifty nine GPs and 3904 patients were recruited. Baseline characteristics of the study sample are presented in Tables 1, 2 and 3, in Additional files 2 and 3. A few patients did not meet the inclusion criteria for the randomised controlled trial (see Table 1), but were included for this analysis. On average, participants were taking 10.5 substances $( \pm 2.4)$ and had 9.5 diagnoses $( \pm 4.9)$. HMG CoA reductase inhibitors were the most commonly used drug, followed by proton pump inhibitors, selective beta blocking agents, platelet aggregation inhibitors, ACE inhibitors, sulphonamides, and dihydropyridine derivatives. Among the ten most commonly used substances according to ATC 5 level, pantoprazole was considered as inappropriate according to the EU(7)-PIM list, and for acetylsalicylic acid, bisoprolol, and amlodipine the EU(7)-PIM list recommended an alternative [32]. Essential (primary) hypertension was the most common diagnosis, followed by disorders of lipoprotein metabolism, type 2 diabetes mellitus, and arthrosis.

\section{Factors associated with excessive polypharmacy}

Table 4 reports the results of the multivariable analysis. Factors were used as described in the methods section. Regarding study centres, Germany 1 as the one having the highest percentage of patients taking $\geq 10$ drugs was chosen as reference for all other centres. Factors significantly associated with excessive polypharmacy were being frail/terminally ill (OR 1.45; 95\% CI 1.22-1.71), having more than 8 diagnoses (OR 2.64; 95\% CI 2.243.11), being obese (OR 1.18; 95\% CI 1.02-1.38), having a lower physical health composite score up to the median (OR 1.47; 95\% CI 1.26-1.72), and a lower mental health composite score up to the median (OR 1.33; 95\% CI $1.17-1.59$ ). In contrast, being $\geq 85$ years old (OR 0.83 ; 95\% CI 0.70-0.99) was significantly associated with a lower risk for excessive polypharmacy. No association with excessive polypharmacy could be found for being female (OR 1.03; 95\% CI 0.89-1.19), having a low educational level (OR 0.95; 95\% CI 0.80-1.13), and being a smoker (OR 0.85; 95\% CI 0.60-1.21). Regarding the study centres, being recruited in the UK led to a significantly higher risk for excessive polypharmacy compared to being recruited in Germany 1 (OR 1.71; 95\% CI 1.272.30). Being recruited in Germany 2 led to a slightly significant lower risk for excessive polypharmacy compared to Germany 1 (OR $0.74 ; 95 \%$ CI $0.56-0.97$ ). There were no significant associations between recruitment in Austria and Germany 1 (OR 0.97; 95\% CI 0.72-1.31) or Italy and Germany 1 (OR 0.90; 95\% CI 0.67-1.21).

\section{Discussion}

This cross-sectional study is based on a large sample of older patients with polypharmacy recruited for the European randomised controlled multicentre trial PRIMA-eDS. Results suggest that frailty, multimorbidity, and obesity as well as lower physical and mental health composite scores on the SF-12 are independent risk factors for excessive polypharmacy. Also, in multivariable analysis, the country or even the region plays an important role. Old age alone ( $\geq 85$ years) does not seem to increase the risk of polypharmacy and may even be associated with lower risk. In our study sample, sex, educational level, and smoking status apparently do not contribute to excessive polypharmacy.

\section{Interpretation and comparison with existing literature}

It is easily understandable that $>8$ diagnoses contribute to excessive polypharmacy because guideline-adherent treatment of multiple diseases will inevitably lead to a large number of drugs being prescribed. This has also been shown by other studies with no polypharmacy as a comparison to excessive polypharmacy [20, 21, 24].

In our study, being frail/terminally ill is significantly associated with excessive polypharmacy. Saum et al. [33] and Herr et al. [34] showed that taking $\geq 10$ drugs compared to taking $\leq 4$ drugs is significantly associated with frailty [24]. Morley et al. [35] described frailty as a risk factor for a medication increase as physicians lack a concept on how to treat frail old people and thus often start medication. However, the question of causality remains unsettled as polypharmacy may also lead to frailty [36], and polypharmacy as well as frailty may be a result of multimorbidity. Studies suggested that there is a dose-response relationship between the number of drugs taken and the risk of being frail $[33,37]$. 
Table 1 Demographic and clinical characteristics of the population

\begin{tabular}{|c|c|c|c|c|c|c|}
\hline \multirow[t]{2}{*}{ Characteristics } & \multicolumn{2}{|c|}{ All subjects $(n=3904)$} & \multicolumn{2}{|c|}{$\begin{array}{l}\text { Polypharmacy 7-10 } \\
\text { substances }^{\mathrm{b}}(n=1644)\end{array}$} & \multicolumn{2}{|c|}{$\begin{array}{l}\text { Excessive Polypharmacy } \geq 10 \\
\text { substances }(n=2260)\end{array}$} \\
\hline & \multicolumn{2}{|l|}{$\bar{n}$} & \multicolumn{2}{|l|}{$\bar{n}$} & \multicolumn{2}{|l|}{$\mathrm{n}$} \\
\hline \multicolumn{7}{|l|}{ Sociodemographic data } \\
\hline Age & 3904 & & & & & \\
\hline $75-85^{\complement}(n, \%)$ & 3036 & 77.8 & 1270 & 77.3 & 1766 & 78.1 \\
\hline$\geq 85(n, \%)$ & 868 & 22.2 & 374 & 22.7 & 494 & 21.9 \\
\hline mean $\pm \mathrm{SD}$ (years) & 3904 & $81.5 \pm 4.4$ & 1644 & $81.6 \pm 4.4$ & 2260 & $81.5 \pm 4.4$ \\
\hline Sex & 3904 & & & & & \\
\hline Female, n (\%) & 2240 & 57.4 & 913 & 55.5 & 1327 & 58.7 \\
\hline Male, n (\%) & 1664 & 42.6 & 731 & 44.5 & 933 & 41.3 \\
\hline Educational level, n (\%) & $3578^{a}$ & & & & & \\
\hline Low & 1536 & 39.3 & 669 & 40.7 & 867 & 38.4 \\
\hline Medium & 1465 & 37.5 & 583 & 35.5 & 882 & 39.0 \\
\hline High & 577 & 14.8 & 239 & 14.5 & 338 & 15.0 \\
\hline Health-related factors & $3736^{a}$ & & & & & \\
\hline Smokers, n (\%) & 154 & 3.9 & 72 & 4.4 & 82 & 3.6 \\
\hline BMI, n (\%) & 3904 & & & & & \\
\hline $\mathrm{BMI}<18.5$ & 34 & 0.9 & 15 & 0.9 & 19 & 0.8 \\
\hline BMI 18.5-24 & 957 & 24.5 & 435 & 26.5 & 522 & 23.1 \\
\hline BMI 25-29 & 1606 & 41.1 & 710 & 43.2 & 896 & 39.7 \\
\hline $\mathrm{BMI} \geq 30$ & 1307 & 33.5 & 484 & 29.4 & 823 & 36.4 \\
\hline Frailty level, n (\%) & $3781^{a}$ & & & & & \\
\hline Managing well & 1643 & 42.1 & 804 & 48.9 & 839 & 37.1 \\
\hline Vulnerable & 868 & 22.2 & 358 & 21.8 & 510 & 22.6 \\
\hline Mildly frail & 660 & 16.9 & 237 & 14.4 & 423 & 18.7 \\
\hline Moderately frail & 505 & 13.0 & 153 & 9.3 & 352 & 15.6 \\
\hline Severely frail & 97 & 2.5 & 32 & 2.0 & 65 & 2.9 \\
\hline Very severely frail & 8 & 0.2 & 2 & 0.1 & 6 & 0.2 \\
\hline Physical health composite score, median (range) & $3484^{\mathrm{a}}$ & $36.6(10-68)$ & 1454 & $39.4(12-68)$ & 2030 & $34.7(10-63)$ \\
\hline Mental health composite score, median (range) & $3483^{\mathrm{a}}$ & $48.7(12-76)$ & 1454 & $50.3(14-76)$ & 2029 & $47.4(12-74)$ \\
\hline Study centre, n (\%) & 3904 & & & & & \\
\hline Austria & 587 & 15.0 & 259 & 15.8 & 328 & 14.5 \\
\hline Germany 1 & 981 & 25.1 & 351 & 21.3 & 630 & 27.9 \\
\hline Germany 2 & 742 & 19.0 & 334 & 20.3 & 408 & 18.1 \\
\hline Italy & 901 & 23.4 & 439 & 26.7 & 462 & 20.4 \\
\hline UK & 693 & 17.8 & 261 & 15.9 & 432 & 19.1 \\
\hline Substances, $\mathrm{n}($ mean $\pm \mathrm{SD})$ & 3904 & $10.5 \pm 2.4$ & 1644 & $8.5 \pm 0.6$ & 2260 & $12.0 \pm 2.2$ \\
\hline Diagnoses, n (mean $\pm \mathrm{SD})$ & $3898^{\mathrm{a}}$ & $9.5 \pm 4.9$ & 1644 & $8.2 \pm 4.2$ & 2260 & $10.5 \pm 5.2$ \\
\hline
\end{tabular}

${ }^{a}$ As $n$ differs from 3904 , the rest of the patients have missing data regarding the variable

b7 patients took $<7$ substances

${ }^{c_{5}}$ patients were $<75$ years old

Legend: $B M I$ body mass index, Germany 1 Rostock, Germany 2 = Witten, SD Standard deviation

Regarding health-related quality of life, a lower physical and mental health composite score indicating worse functioning within these health domains are significantly associated with excessive polypharmacy. Jyrkka et al. found moderate and poor self-reported health to be risk factors for excessive polypharmacy compared to no polypharmacy [20]. Polypharmacy patients usually suffer from several diseases and we expect them to have a 
Table 2 Percentage of the population using substances (ATC level 4) according to polypharmacy status

\begin{tabular}{|c|c|c|c|c|c|c|c|}
\hline \multicolumn{2}{|c|}{ Substances } & \multicolumn{2}{|c|}{ All subjects } & \multicolumn{2}{|c|}{ Polypharmacy $<10$ substances } & \multicolumn{2}{|c|}{ Excessive polypharmacy $\geq 10$ substances } \\
\hline & & $\mathrm{n}$ & (\%) & $\mathrm{n}$ & (\%) & $\mathrm{n}$ & (\%) \\
\hline C10AA & HMG CoA reductase inhibitors & 2479 & 63.5 & 999 & 60.8 & 1480 & 65.5 \\
\hline $\mathrm{A} 02 \mathrm{BC}$ & Proton pump inhibitors & 2328 & 59.6 & 837 & 50.9 & 1491 & 66.0 \\
\hline CO7AB & Beta blocking agents, selective & 2240 & 57.4 & 909 & 55.3 & 1331 & 58.9 \\
\hline B01AC & Platelet aggregation inhibitors excl. Heparin & 1952 & 50.0 & 762 & 46.4 & 1190 & 52.7 \\
\hline CO9AA & ACE Inhibitors, plain & 1751 & 44.9 & 792 & 48.2 & 959 & 42.4 \\
\hline C03CA & Sulfonamides, plain & 1715 & 43.9 & 569 & 34.6 & 1146 & 50.7 \\
\hline C08CA & Dihydropyridine derivates & 1611 & 41.3 & 658 & 40.0 & 953 & 42.2 \\
\hline CO9CA & Angiotensin II antagonists, plain & 1355 & 34.7 & 505 & 30.7 & 850 & 37.6 \\
\hline CO3AA & Thiazides, plain & 1354 & 34.7 & 555 & 33.8 & 799 & 35.4 \\
\hline A11CC & Vitamin D and analogues & 1120 & 28.7 & 345 & 21.0 & 775 & 34.3 \\
\hline
\end{tabular}

reduced health-related quality of life due to illness. Here again, causality may not be easily determined as polypharmacy could also lead to a decrease in health-related quality of life e.g. due to adverse effects of drugs.

It is not surprising that obesity is associated with excessive polypharmacy. Obesity has been shown to lead to an increased use of drugs [38] and can result in chronic diseases especially with advancing age [39]. However, a causal inference cannot be made as chronic diseases caused by obesity may be associated with excessive polypharmacy and it might be that obesity is an intermediate variable or a confounder.

We found that being $\geq 85$ years of age is a protective factor against excessive polypharmacy. This was also found by Kim et al. [21] when comparing older patients with and without polypharmacy, and by Onder et al. [24] who detected an inverse correlation between polypharmacy and increasing age. However, Jyrkka et al. [20] found $\geq 85$ years to be a risk factor for excessive polypharmacy compared to no polypharmacy, and two further studies [19, 25] showed being $\geq 80$ years of age to be a risk factor. One explanation for the decreased use of drugs might be that due to a limited life expectancy of these very old people preventive medications are stopped in order to improve the patients' current well-being [40]. However, whether this really happens is questionable. Age does not influence patients' priorities in taking preventive medication and reducing adverse events [41], and GPs find deprescribing of preventive medication difficult [42]. Another interpretation could be that excessive polypharmacy patients die earlier and do not reach the very old age.

We did not find that sex was significantly associated with excessive polypharmacy. The literature is conflicting here $[19,20,24,25]$. In this study there was no significant association between educational level and excessive polypharmacy. In the literature it has been shown that educational level had an impact on health, however, this effect appeared to decrease with age and was not significant anymore in adults $\geq 51$ years [43].

Smoking contributes to the burden of disease. Surprisingly, smoking was not associated with excessive

Table 3 Most common diagnoses of the population according to polypharmacy status

\begin{tabular}{|c|c|c|c|c|c|c|c|}
\hline \multicolumn{2}{|c|}{ Diagnoses } & \multicolumn{2}{|c|}{ All subjects } & \multicolumn{2}{|c|}{$\begin{array}{l}\text { Polypharmacy } \\
<10 \text { substances }\end{array}$} & \multicolumn{2}{|c|}{$\begin{array}{l}\text { Excessive polypharmacy } \\
\geq 10 \text { substances }\end{array}$} \\
\hline & & \multirow{2}{*}{$\begin{array}{l}\mathrm{n} \\
3428\end{array}$} & \multirow{2}{*}{$\frac{(\%)}{87.8}$} & \multirow{2}{*}{$\begin{array}{l}n \\
1426\end{array}$} & \multirow{2}{*}{$\frac{(\%)}{86.7}$} & \multirow{2}{*}{$\begin{array}{l}n \\
2002\end{array}$} & \multirow{2}{*}{$\begin{array}{l}\%) \\
88.6\end{array}$} \\
\hline 110 & Essential (primary) hypertension & & & & & & \\
\hline E78 & Disorders of lipoprotein metabolism and other lipidaemias & 2078 & 53.2 & 814 & 49.5 & 1264 & 55.9 \\
\hline E11 & Type 2 diabetes mellitus & 1850 & 47.4 & 686 & 41.7 & 1164 & 51.5 \\
\hline M19 & Osteoarthritis & 1752 & 44.9 & 683 & 41.6 & 1069 & 47.3 \\
\hline 125 & Chronic ischaemic heart disease & 1473 & 37.7 & 566 & 34.4 & 907 & 40.1 \\
\hline M54 & Dorsalgia & 1442 & 36.9 & 499 & 30.4 & 943 & 41.7 \\
\hline 148 & Atrial fibrillation and flutter & 1172 & 30.0 & 471 & 28.7 & 701 & 31.0 \\
\hline 150 & Heart failure & 1142 & 29.3 & 412 & 25.1 & 730 & 32.3 \\
\hline K21 & Gastro-oesophageal reflux disease & 982 & 25.2 & 369 & 22.5 & 613 & 27.1 \\
\hline F32 & Depressive episode & 853 & 21.9 & 292 & 17.8 & 561 & 24.8 \\
\hline
\end{tabular}


Table 4 Factors associated with excessive polypharmacy ( $\geq 10$ substances); results from the multivariable generalized linear mixed model

\begin{tabular}{|c|c|c|c|c|}
\hline \multirow[b]{2}{*}{ Factors } & \multicolumn{2}{|l|}{ Univariable } & \multicolumn{2}{|l|}{ Multivariable } \\
\hline & $\mathrm{OR}(95 \% \mathrm{Cl})$ & $p$ & $\mathrm{OR}(95 \% \mathrm{Cl})$ & $p$ \\
\hline Sex (female vs male) & $1.18(1.03-1.35)$ & 0.0189 & $1.03(0.89-1.19)$ & 0.6718 \\
\hline Age group ( $\geq 85$ vs < 85) & $0.96(0.82-1.13)$ & 0.6388 & $0.83(0.70-0.99)$ & 0.0328 \\
\hline Educational level (low vs medium/high/missing) & $0.94(0.81-1.10)$ & 0.4440 & $0.95(0.80-1.13)$ & 0.5506 \\
\hline Frailty (frail/terminally ill vs managing well/vulnerable/missing) & $1.83(1.57-2.13)$ & $<.0001$ & $1.45(1.22-1.71)$ & $<.0001$ \\
\hline $\mathrm{BMI}(\geq 30 \mathrm{vs}<30)$ & $1.34(1.16-1.54)$ & $<.0001$ & $1.18(1.02-1.38)$ & 0.0303 \\
\hline Smoker (smoker vs non-smoker/ex-smoker/missing) & $0.83(0.59-1.16)$ & 0.2763 & $0.85(0.60-1.21)$ & 0.3579 \\
\hline Research centre (Austria vs Germany 1) & $0.69(0.52-0.93)$ & 0.0139 & $0.97(0.72-1.31)$ & 0.8519 \\
\hline Research centre (Italy vs Germany 1) & $0.58(0.44-0.75)$ & $<.0001$ & $0.90(0.67-1.21)$ & 0.4950 \\
\hline Research centre (UK vs Germany 1) & $0.92(0.70-1.22)$ & 0.5561 & $1.71(1.27-2.30)$ & 0.0004 \\
\hline Research centre (Germany 2 vs Germany1) & $0.71(0.54-0.94)$ & 0.0150 & $0.74(0.56-0.97)$ & 0.0303 \\
\hline Number of diagnoses (> 8 vs $\leq 8$ ) & $2.70(2.32-3.14)$ & $<.0001$ & $2.64(2.24-3.11)$ & $<.0001$ \\
\hline Physical health composite score ( $\leq 36.635$ vs $>36.636$ ) & $1.83(1.58-2.11)$ & $<.0001$ & $1.47(1.26-1.72)$ & $<.0001$ \\
\hline Mental health composite score ( $\leq 48.7$ vs $>48.8$ ) & $1.53(1.33-1.76)$ & $<.0001$ & $1.36(1.17-1.59)$ & $<.0001$ \\
\hline
\end{tabular}

Legend: Germany 1 = Rostock, Germany 2 = Witten

polypharmacy in older polypharmacy patients, but there were very few smokers among the patients in our study.

There was a slightly significant association between excessive polypharmacy and the study centre Germany 1 when compared to Germany 2. One possible explanation could be the differences between the two settings. Germany 1 recruited patients in a more rural setting in the former Eastern part of Germany while Germany 2 recruited patients in the large metropolitan area of the highly industrialised Ruhr-region. These differences cannot be explained by the variables recorded in this study and deserve further investigation. The study centre in the UK was significantly associated with excessive polypharmacy compared to the study centre Germany 1 in the multivariable analysis. A sensitivity analysis showed that the UK was significantly associated with excessive polypharmacy compared to all other centres. Interestingly, the univariable analysis showed a slightly divergent result which was not significant (OR 0.92; 95\% CI 0.70-1.22).

The patients in Germany 1 seemed to be frailer and had more diagnoses compared to the patients in the UK. In multivariable analysis the UK resulted in having more excessive polypharmacy. A reversal of effect can result due to the adjustment in the multivariable model. A possible explanation could be the "Quality and Outcomes Framework" (QOF) introduced in 2004 in the UK, which set financial incentives for certain performance indicators (pay-for-performance). Among these were indicators that relate to chronic conditions [44], some of them directly naming the prescription of certain drugs while other indicators indirectly entailed drug treatment in order to reach the targets [45]. Studies observed rising prescription rates of drugs indicated by QOF around the time when the framework was implemented, such as lipid-regulating drugs, renin-angiotensin system drugs [45], ß-blockers or antiplatelet therapy [46].

\section{Implications}

Understanding the health characteristics of an aged population taking several drugs, and investigating factors influencing excessive polypharmacy is highly relevant in times when the geriatric population is growing. This study helps to develop targeted strategies to reduce polypharmacy by identifying factors contributing to excessive polypharmacy. Physicians should especially pay attention to their frail, obese patients that have $>8$ diagnoses, check whether all medications are necessary, evidence based and appropriate, and whether there are relevant interactions. To do so, GPs should perform medication reviews for their patients with excessive polypharmacy on a regular basis to optimise these patients' medication. They should allocate extra time to care for these complex patients which needs to be reimbursed by the health care system.

\section{Strengths and limitations}

The major strength of our study is that we examined a very large sample of older patients representing several different health care settings/countries. We collected various parameters in this geriatric study population which gave us a comprehensive overview of demographic, clinical and functional status, and recorded the frailty level to distinguish between the fitter and the less fit ones. A major limitation of our study is that its cross-sectional design does not allow conclusions on causality. Further limitations are that the health-related quality of life was self-reported and all variables in the eCRF were reported by the GP, by practice staff or by a clinical research nurse. Even though instructions to 
record patient data were the same throughout all settings, we do not know whether the documentation of variables differs in different settings. True drug consumption is difficult to assess. We instructed the GPs to talk to their patients about all drugs they are taking. However, we were not able to verify drug consumption. Also, in this cross-sectional analysis, only patients were analysed who were recruited according to the inclusion criteria of the PRIMA-eDS trial. We therefore could only investigate patient characteristics associated with excessive polypharmacy in comparison to less excessive polypharmacy as patients without polypharmacy were not included in the trial. Furthermore, external characteristics e.g. of the prescribers could not be taken into account, and we did not judge whether medication intake was appropriate or not.

A further limitation of this study is that multiple relationships between variables exist. Multicollinearity is the cause of conspicuous differences between univariable and multivariable analysis. The interpretation for the affected variables should be regarded with caution. Noticeable correlations were found for the relationship between the research centre and the educational level (Cramer's $\mathrm{V}=0.48$ ), or the number of diagnoses (Cramer's $\mathrm{V}=0.40$ ) respectively, as well as between frailty, the two SF-12 scales (physical health composite score Cramer's $V=0.20$ and mental health composite score Cramer's V $=0.33$ ) and age (Cramer's V $=0.22)$.

The physical and the mental health composite scores as well as frailty were identified as risk factors in both univariable and multivariable analysis, but the ORs are smaller in multivariable analysis because of the dependencies. Frailty and health-related quality of life are closely associated [47], still we retained these variables in our analysis as they measure different concepts.

In univariable analysis, age was not significantly related to polypharmacy. It could be that there is a connection of the variable age with information about the condition of the patient, such as frailty, health-related quality of life, and diagnoses. Frailty [48], lower health-related quality of life [49], and a high number of diagnoses [50] are more common in old age. On the other hand, these factors increase the likelihood of excessive polypharmacy regardless of age $[33,50,51]$. Therefore, the positive effect of high age may become more apparent when adjusting for these factors.

Problematic is the variable "research centre", which significantly increases the associations found after adjustment for UK/Manchester. This is mainly attributable to the consideration of the number of diagnoses. After adjustment for this variable, the OR increases from 1.10 to 1.71 . We did not want to give up the number of diagnoses as an independent variable, as in the literature this is reported as an important risk factor. The variable "research centre" also seemed essential for the model, since this variable represents a variety of influences, such as the quality of the data collection, country-specific features and so on. Yet, it must be regarded with caution.

\section{Conclusion}

Our data suggest that frailty, multimorbidity, obesity as well as low physical and mental health status may be risk factors for excessive polypharmacy. Very old age appears to be a protective factor. Sex, educational level, and smoking are not associated with excessive polypharmacy. To avoid excessive polypharmacy with its possibly unfavourable effects, physicians should carefully review the appropriateness of medication, especially in multimorbid, obese and frail patients.

\section{Additional files}

Additional file 1: Electronic Case Report Form (eCRF) (PDF 201 kb) Additional file 2: Demographic and clinical characteristics per study centre (PDF $109 \mathrm{~kb}$ )

Additional file 3: Percentage of the population using substances (ATC level 5) according to polypharmacy status (PDF $41 \mathrm{~kb}$ )

\section{Abbreviations}

ATC: Anatomical Therapeutic Chemical; BMI: Body mass index; Cl: Confidence interval; GP: General practitioner; OR: Odds ratio; PRIMA-eDS: Polypharmacy in chronic diseases: Reduction of Inappropriate Medication and Adverse drug events in older populations by electronic Decision Support; QOF: Quality and Outcomes Framework; SD: Standard deviation; UK: United Kingdom

\section{Acknowledgements}

We would like to express our gratitude to all participating GPs and patients. Furthermore, we would like to thank the PRIMA-eDS team for their support in collecting data.

\section{Funding}

This study is funded by the 7th framework programme of the European Union, theme Health-2012-Innovation-1-2.2.2-2, grant agreement no. 305388-2.

\section{Availability of data and materials}

The datasets used and/or analysed during the current study are available from the corresponding author on reasonable request.

\section{Authors' contributions}

AR and $A S$ conceptualised the study. ED, AE, TJ, SK, CL, IK, JM, GP, AR, and AV were involved in collecting data. RKM and UST performed the analysis. AR drafted the manuscript. ED, AE, TJ, SK, RKM, CL, IK, JM, GP, AS, UST, and AV critically reviewed the manuscript. All authors read and approved the final manuscript.

\section{Ethics approval and consent to participate}

The PRIMA-eDS study has been approved by the five local ethics committees: 1. Ethikkomission der Universität Witten/Herdecke, 3 December 2013, ref. 103/2013; 2. NRES Committee North West Greater Manchester East, 6 June 2014, ref. 14/NW/0197; 3. Ethikkommission für das Bundesland Salzburg, 15 September 2013, ref. 08.04.2014 (415-E/1509/20-2014); 4. Ethikkommission der Universitätsmedizin Rostock, 3 February 2014, ref. A 2014-0020; and 5. Comitato etico di Belluno (Azienda ULSS), 19 June 2013, ref. 305,388-2. Furthermore, all participants provided written informed consent.

Consent for publication

Not applicable.

Competing interests

The authors declare that they have no competing interests. 


\section{Publisher's Note}

Springer Nature remains neutral with regard to jurisdictional claims in published maps and institutional affiliations.

\begin{abstract}
Author details
${ }^{1}$ Institute of General Practice and Family Medicine, Faculty of Health, Witten/ Herdecke University, Alfred-Herrhausen-Str. 50, 58448 Witten, Germany. ${ }^{2}$ Department of Medical Informatics, Biometry and Epidemiology, Ruhr University, Universitätsstr. 105, 44789 Bochum, Germany. ${ }^{3}$ Institute of General Practice, Rostock University Medical Center, Doberaner Str. 142, 18057 Rostock, Germany. ${ }^{4} \mathrm{NIHR}$ School of Primary Care Research, University of Manchester, Oxford Road 176, M13 9PL, Manchester, UK. ${ }^{5}$ Centre for Primary Care, NIHR School of Primary Care Research, University of Manchester, Oxford Road M13 9PL, Manchester, UK. 'Duodecim Medical Publications Ltd., Kaivokatu 10 A, 00100 Helsinki, Finland. South Tyrolean Academy of General Practice, Wangergasse 18, 39100 Bolzano, Italy.
\end{abstract}

Received: 5 March 2018 Accepted: 13 June 2018

Published online: 18 July 2018

\section{References}

1. Eurostat. Population structure and ageing. 2016. http://ec.europa.eu/ eurostat/statistics-explained/index.php/Population_structure_and_ ageing\#Further_Eurostat_information.

2. Halvorsen $\mathrm{KH}$, Selbaek G, Ruths S. Trends in potentially inappropriate medication prescribing to nursing home patients: comparison of three cross-sectional studies. Pharmacoepidemiol Drug Saf. 2017;26:192-200. https://doi.org/10.1002/pds.4142

3. Tsoi CS, Chow JY, Choi KS, Li H-W, Nie JX, Tracy CS, et al. Medical characteristics of the oldest old: retrospective chart review of patients aged 85+ in an academic primary care Centre. BMC Res Notes. 2014;7:340. https://doi.org/10.1186/1756-0500-7-340 .

4. Wauters M, Elseviers M, Vaes B, Degryse J, Dalleur O, Vander Stichele R, et al. Polypharmacy in a Belgian cohort of community-dwelling oldest old (80+). Acta Clin Belg. 2016;71:158-66. https://doi.org/10.1080/17843286.2016. 1148298

5. Junius-Walker U, Theile G, Hummers-Pradier E. Prevalence and predictors of polypharmacy among older primary care patients in Germany. Fam Pract. 2007:24:14-9. https://doi.org/10.1093/fampra/cml067.

6. Herr M, Robine J-M, Pinot J, Arvieu J-J, Ankri J. Polypharmacy and frailty: prevalence, relationship, and impact on mortality in a French sample of 2350 old people. Pharmacoepidemiol Drug Saf. 2015;24:637-46. https://doi. org/10.1002/pds.3772

7. Hovstadius B, Petersson G, Hellström L, Ericson L. Trends in inappropriate drug therapy prescription in the elderly in Sweden from 2006 to 2013: assessment using national indicators. Drugs Aging. 2014;31:379-86. https:// doi.org/10.1007/s40266-014-0165-5

8. Guerriero F, Orlando V, Tari DU, Di Giorgio A, Cittadini A, Trifiro G, Menditto E. How healthy is community-dwelling elderly population? Results from Southern Italy Transl Med UniSa. 2015;13:59-64.

9. Bushardt RL, Massey EB, Simpson TW, Ariail JC, Simpson KN. Polypharmacy: misleading, but manageable. Clin Interv Aging. 2008;3:383-9.

10. Masnoon N, Shakib S, Kalisch-Ellett L, Caughey GE. What is polypharmacy? A systematic review of definitions. BMC Geriatr. 2017;17:230. https://doi.org/10. 1186/s12877-017-0621-2

11. Guthrie B, Makubate B, Hernandez-Santiago V, Dreischulte T. The rising tide of polypharmacy and drug-drug interactions: population database analysis 19952010. BMC Med. 2015;13:74. https://doi.org/10.1186/s12916-015-0322-7

12. Corsonello A, Pedone C, Incalzi RA. Age-related pharmacokinetic and pharmacodynamic changes and related risk of adverse drug reactions. Curr Med Chem. 2010:17:571-84.

13. Fried TR, O'Leary J, Towle V, Goldstein MK, Trentalange M, Martin DK. Health outcomes associated with polypharmacy in community-dwelling older adults: a systematic review. J Am Geriatr Soc. 2014;62:2261-72. https://doi. org/10.1111/jgs.13153

14. Agostini JV, Han L, Tinetti ME. The relationship between number of medications and weight loss or impaired balance in older adults. J Am Geriatr Soc. 2004;52:1719-23. https://doi.org/10.1111/j.1532-5415.2004.52467.x

15. Wimmer BC, Cross AJ, Jokanovic N, Wiese MD, George J, Johnell K, et al. Clinical outcomes associated with medication regimen complexity in older people: a systematic review. J Am Geriatr Soc. 2017;65:747-53. https://doi. org/10.1111/jgs.14682

16. Payne RA, Abel GA, Avery AJ, Mercer SW, Roland MO. Is polypharmacy always hazardous? A retrospective cohort analysis using linked electronic health records from primary and secondary care. Br J Clin Pharmacol. 2014; 77:1073-82. https://doi.org/10.1111/bcp.12292

17. The King's Fund. Polypharmacy and medicines optimisation: Making it safe and sound. 2013.

18. Corsonello A, Pedone C, Corica F, Incalzi RA. On behalf of the Gruppo Italiano di. Polypharmacy in elderly patients at discharge from the acute care hospital. Ther Clin Risk Manag. 2007;3:197-203. https://doi.org/10.2147/ tcrm.2007.3.1.197.

19. Haider SI, Johnell K, Weitoft GR, Thorslund M, Fastbom J. The influence of educational level on polypharmacy and inappropriate drug use: a register-based study of more than 600,000 older people. J Am Geriatr Soc. 2009;57:62-9. https://doi.org/10.1111/j.1532-5415.2008. 02040.x

20. Jyrkka J, Enlund H, Korhonen MJ, Sulkava R, Hartikainen S. Patterns of drug use and factors associated with polypharmacy and excessive polypharmacy in elderly persons: results of the Kuopio 75+ study: a cross-sectional analysis. Drugs Aging. 2009:26:493-503. https://doi.org/10.2165/00002512200926060-00006

21. Kim H-A, Shin J-Y, Kim M-H, Park B-J. Prevalence and predictors of polypharmacy among Korean elderly. PLoS One. 2014;9:e98043. https://doi. org/10.1371/journal.pone.0098043

22. Mayer S, Osterle A. Socioeconomic determinants of prescribed and nonprescribed medicine consumption in Austria. Eur J Pub Health. 2015;25:597603. https://doi.org/10.1093/eurpub/cku179

23. O'Dwyer M, Peklar J, McCallion P, McCarron M, Henman MC. Factors associated with polypharmacy and excessive polypharmacy in older people with intellectual disability differ from the general population: a crosssectional observational nationwide study. BMJ Open. 2016:6:e010505. https://doi.org/10.1136/bmjopen-2015-010505

24. Onder G, Liperoti R, Fialova D, Topinkova E, Tosato M, Danese $P$, et al. Polypharmacy in nursing home in Europe: results from the SHELTER study. J Gerontol A Biol Sci Med Sci. 2012;67:698-704. https://doi.org/10.1093/gerona/glr233.

25. Walckiers D, van der Heyden J, Tafforeau J. Factors associated with excessive polypharmacy in older people. Arch Public Health. 2015;73:50. https://doi.org/10.1186/s13690-015-0095-7.

26. Sonnichsen A, Trampisch US, Rieckert A, Piccoliori G, Vogele A, Flamm M, et al. Polypharmacy in chronic diseases-reduction of inappropriate medication and adverse drug events in older populations by electronic decision support (PRIMA-eDS): study protocol for a randomized controlled trial. Trials. 2016;17:57. https://doi.org/10.1186/s13063-016-1177-8

27. Rockwood K, Song X, MacKnight C, Bergman H, Hogan DB, McDowell I, Mitnitski A. A global clinical measure of fitness and frailty in elderly people. CMAJ. 2005:173:489-95. https://doi.org/10.1503/cmaj.050051

28. Maruish MEE. User's manual for the SF-12v2 health survey. 3rd ed. Lincoln RI: QualityMetric Incorporated; 2012.

29. OECD. Classifying Educational Programmes - Manual for ISCED-97 Implementation in OECD Countries - 1999 Edition. OECD. 1999

30. World Health Organization. The Anatomical Therapeutic Chemical Classification System with Defined Daily Doses (ATC/DDD). http://www.who. int/classifications/atcddd/en/. Accessed 14 Feb 2018.

31. World Health Organization. Classification of Diseases (ICD). http://www.who int/classifications/icd/en/. Accessed 14 Feb 2018.

32. Renom-Guiteras A, Meyer G, Thürmann PA. The EU(7)-PIM list: a list of potentially inappropriate medications for older people consented by experts from seven European countries. Eur J Clin Pharmacol. 2015;71:86175. https://doi.org/10.1007/s00228-015-1860-9

33. Herr M, Sirven N, Grondin H, Pichetti S, Sermet C. Frailty, polypharmacy, and potentially inappropriate medications in old people: findings in a representative sample of the French population. Eur J Clin Pharmacol. 2017; https://doi.org/10.1007/s00228-017-2276-5.

34. Saum K-U, Schöttker B, Meid AD, Holleczek B, Haefeli WE, Hauer K, Brenne HI. Polypharmacy associated with frailty in older people? Results from the ESTHER cohort study. J Am Geriatr Soc. 2017;65:e27-32. https://doi.org/10. 1111/jgs. 14718.

35. Morley JE. Inappropriate drug prescribing and polypharmacy are major causes of poor outcomes in long-term care. J Am Med Dir Assoc. 2014;15: 780-2. https://doi.org/10.1016/j.jamda.2014.09.003 
36. Morley JE, Vellas B, van Kan GA, Anker SD, Bauer JM, Bernabei R, et al. Frailty consensus: a call to action. J Am Med Dir Assoc. 2013;14:392-7. https://doi. org/10.1016/j.jamda.2013.03.022 .

37. Veronese N, Stubbs B, Noale M, Solmi M, Pilotto A, Vaona A, et al. Polypharmacy is associated with higher frailty risk in older people: an 8-year longitudinal cohort study. J Am Med Dir Assoc. 2017;18:624-8. https://doi. org/10.1016/j.jamda.2017.02.009

38. Counterweight Project Team. The impact of obesity on drug prescribing in primary care. Br J Gen Pract. 2005;55:743-9.

39. Amarya S, Singh K, Sabharwal M. Health consequences of obesity in the elderly. J Clinical Gerontology Geriatrics. 2014;5:63-7. https://doi.org/10. 1016/j.jcgg.2014.01.004

40. Lee SJ, Leipzig RM, Walter LC. Incorporating lag time to benefit into prevention decisions for older adults. JAMA. 2013;310:2609-10. https://doi. org/10.1001/jama.2013.282612

41. Tinetti ME, McAvay GJ, Fried TR, Allore HG, Salmon JC, Foody JM, et al. Health outcome priorities among competing cardiovascular, fall injury, and medication-related symptom outcomes. J Am Geriatr Soc. 2008;56:1409-16. https://doi.org/10.1111/j.1532-5415.2008.01815.x

42. Schuling J, Gebben H, Veehof LJG, Haaijer-Ruskamp FM. Deprescribing medication in very elderly patients with multimorbidity: the view of Dutch GPs. A qualitative study. BMC Fam Pract. 2012;13:56. https://doi.org/10.1186/ 1471-2296-13-56 .

43. Baker DP, Leon J, Smith Greenaway EG, Collins J, Movit M. The education effect on population health: a reassessment. Popul Dev Rev. 2011;37:307-32. https://doi.org/10.1111/j.1728-4457.2011.00412.x

44. Roland M. Linking physicians' pay to the quality of care-a major experiment in the United Kingdom. N Engl J Med. 2004;351:1448-54. https://doi.org/10. 1056/NEJMhpr041294

45. MacBride-Stewart SP, Elton R, Walley T. Do quality incentives change prescribing patterns in primary care? An observational study in Scotland. Fam Pract. 2008;25:27-32. https://doi.org/10.1093/fampra/cmm074 .

46. McGovern MP, Boroujerdi MA, Taylor MW, Williams DJ, Hannaford PC, Lefevre KE, Simpson CR. The effect of the UK incentive-based contract on the management of patients with coronary heart disease in primary care. Fam Pract. 2008;25:33-9. https://doi.org/10.1093/fampra/cmm073.

47. Kojima G, lliffe S, Jivraj S, Walters K. Association between frailty and quality of life among community-dwelling older people: a systematic review and meta-analysis. J Epidemiol Community Health. 2016;70:716-21. https://doi. org/10.1136/jech-2015-206717

48. Frailty, polypharmacy and deprescribing. Drug Ther Bull 2016;54:69-72. doi: https://doi.org/10.1136/dtb.2016.6.0408 .

49. Eisele M, Kaduszkiewicz H, König H-H, Lange C, Wiese B, Prokein J, et al. Determinants of health-related quality of life in older primary care patients: results of the longitudinal observational AgeCoDe study. Br J Gen Pract. 2015;65:e716-23. https://doi.org/10.3399/bjgp15X687337.

50. Puth M-T, Weckbecker K, Schmid M, Münster E. Prevalence of multimorbidity in Germany: impact of age and educational level in a crosssectional study on 19,294 adults. BMC Public Health. 2017;17:826. https://doi. org/10.1186/s12889-017-4833-3.

51. Montiel-Luque A, Núñez-Montenegro AJ, Martín-Aurioles E, Canca-Sánchez JC, Toro-Toro MC, González-Correa JA. Medication-related factors associated with health-related quality of life in patients older than 65 years with polypharmacy. PLoS One. 2017; https://doi.org/10.1371/journal.pone. 0171320

\section{Ready to submit your research? Choose BMC and benefit from:}

- fast, convenient online submission

- thorough peer review by experienced researchers in your field

- rapid publication on acceptance

- support for research data, including large and complex data types

- gold Open Access which fosters wider collaboration and increased citations

- maximum visibility for your research: over $100 \mathrm{M}$ website views per year

At BMC, research is always in progress.

Learn more biomedcentral.com/submissions 